\title{
Diagnostic Procedures for Lung Cancer According to Histological Types and Their Complications
}

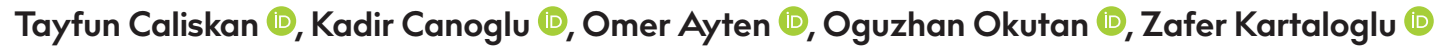 \\ Department of Pulmonology, Sultan Abdulhamid Han Training and Research Hospital, Health Sciences University, İstanbul, Turkey \\ ORCID iDs of the authors: T.C. 0000-0002-7905-2430; K.C. 0000-0003-1579-3392; 0.A. 0000-0002-2275-4378; 0.0. 0000-0002-4660- \\ I595; Z.K. 0000-0002-2954-6168.
}

Cite this article as: Caliskan T, Canoglu K, Ayten O, Okutan O, Kartaloglu Z. Diagnostic Procedures for Lung Cancer According to Histological Types and Their Complications. Cyprus J Med Sci 202I; 6(I): 3-7.

\section{BACKGROUND/AIMS}

This study aimed to investigate if there are any differences between the diagnostic methods used in biopsy for lung cancer on the basis of the histological cell types. In addition, the contribution of bronchoscopic washing in the diagnosis of lung cancer and complications of flexible bronchoscopy and computed tomography (CT)-guided transthoracic needle biopsy (TNB) were also investigated.

\section{MATERIAL and METHODS}

The patients who were diagnosed with lung cancer pathologically were divided into two groups-bronchoscopic biopsy and CT-guided TNB. A comparison was made between the two groups according to the lung cancer cell type. Complication rates for both procedures were calculated. In addition, the contribution of bronchoscopic washing in the diagnosis of lung cancer was also investigated in patients who underwent bronchoscopy.

\section{RESULTS}

The study included 182 lung cancer patients, out of whom 106 patients were diagnosed by bronchoscopy and 76 patients were diagnosed by CT-guided TNB. There was no significant difference between the diagnostic approaches for adenocarcinoma (ADC) ( $p=.22)$. Small cell lung cancer (SCLC) and squamous cell carcinoma (SCC) were diagnosed more accurately by bronchoscopy than TNB ( $p=.01, p=.01$, respectively). Bronchoscopic washing was found to be positive in 3 of the patients who had negative results with bronchoscopic biopsy. There were no major complications related to bronchoscopy. The total minor complication rate of bronchoscopy was found to be $11.3 \%$. Pneumothorax was detected in $17 \%$ of patients undergoing TNB and the total complication rate of TNB was $19.7 \%$.

\section{CONCLUSION}

SCLC and SCC were more accurately diagnosed by flexible bronchoscopy than TNB. Bronchoscopic washing contributed in the diagnosis of lung cancers. Flexible bronchoscopy was found to be much safer and associated with lower complication rates than CT-guided TNB.

Keywords: Bronchoscopy, transthoracic needle biopsy, bronchial washing, lung cancer.

\section{INTRODUCTION}

Lung cancer is the leading cause of cancer deaths among both men and women (I). Fast and accurate detection and staging are important in order to decide most appropriate treatment for patients with lung cancer. Flexible bronchoscopy (FB) and computed tomography (CT)-guided transthoracic needle biopsy (TNB) are used for the diagnosis of lung cancer on the basis of the localization of the lesion in the lungs. TNB is used especially for peripheral pulmonary nodules, and approximately $90 \%$ of patients with pulmonary nodules can be diagnosed with TNB (2). However, pneumothorax is detected in approximately $25 \%$ of the patients who underwent TNB (3). Chest tube is required for the treatment of $15 \%$ of the patients with pneumothorax (3). Conventional FB has a diagnostic accuracy of $64 \%$ in malignant lesions and $35 \%$ in benign lesions (4). The diagnostic accuracy of FB in central, middle, and peripherally located lesions is $82 \%, 61 \%$, and $53 \%$, respectively, when lesions are classified on the basis of localization (4). Diagnostic value of FB decreases when localization of the lesion changes from central to peripheral. Bronchoscopic biopsy can be used with bronchial washing, brushing, and transbronchial needle biopsy to achieve a diagnostic yield between $65 \%$ and $88 \%$ (5). FB has been shown to be a safer procedure and associated with lesser complications such as mortality, bleeding, 
and pneumothoraxes that necessitate intervention (6). FB is an ideal technique for large, central lesions and diagnostic yields decrease between $30 \%$ and $40 \%$ without an endobronchial lesion. Bronchial washing contributes to bronchoscopy in the diagnosis of lung cancer, and it was found positive in $40 \%$ of the patients with pulmonary nodules (4). Lung cancers are detected in different locations within the lung and are classified on the basis of the histological cell types. Small cell lung cancer (SCLC) is usually centrally located (7); adenocarcinoma (ADC) is a histologically heterogeneous peripheral tumor; squamous cell carcinoma (SCC) is typically detected as a central endobronchial mass; and large cell carcinoma (LCC) is seen as poorly differentiated large peripheral masses.

This study aimed to investigate whether there is a difference between the diagnostic methods used in biopsy for lung cancer according to cell type. In addition, the contribution of bronchoscopic washing in the diagnosis of lung cancer and biopsy complications of FB and TNB were studied.

\section{MATERIAL and METHODS}

Patients who were diagnosed with lung cancer pathologically by biopsy between January I, 2018 and October I, 2019 were included in the study. The study was conducted retrospectively, and an approval was obtained from the local ethics committee.

FB was performed in conscious patients. Routine vital signs such as arterial blood pressure, heart rate and rhythm, and oxygen saturation $\left(\mathrm{SpO}_{2}\right)$ were recorded during FB. All patients were monitored for symptoms including dyspnea, chest pain, and hemoptysis after completion of FB. Oxygen was administered through a nasal cannula to ensure that $\mathrm{SpO}_{2}$ was between $91 \%$ and $93 \%$. For oropharyngeal topical anesthesia, $10 \%$ lidocaine spray was used. Midazolam ( $-5 \mathrm{mg}$ ) was used as a sedative for FB. For topical anesthesia, 2\% lidocaine solution via the bronchoscope working channel was used. Complications associated with bronchoscopy were reported as procedure-related complications (hypoxemia, hemorrhage, fever, pneumothorax, bronchospasm, laryngospasm, pneumonia, and death) and sedation- and anesthesia-related complications (hypoxemia, respiratory depression, hypotension, hypertension, syncope, arrhythmias, seizures, methemoglobinemia, and death). If hemorrhage was $>200 \mathrm{~mL} / \mathrm{d}$, it was recorded

\section{Main Points:}

- Small cell lung carcinoma and squamous cell carcinoma was diagnosed more accurately with flexible bronchoscopy. There was no difference between the two diagnostic methods for adenocarcinoma.

- There were no major complications associated with flexible bronchoscopy; however, minor complications developed in $11.3 \%$ of the patients. Pneumothorax was detected in $17 \%$ of the patients who underwent computed tomography (CT)-guided transthoracic needle biopsy (TNB), and the total complication rate was found to be 19.73\% for CT-guided TNB.

- Flexible bronchoscopy was found to be a relatively safe diagnostic method with a lower complication rate compared to CT-guided TNB in the diagnosis of lung cancer. as a major hemorrhage, otherwise, it was recorded as a minor hemorrhage. Death or complications requiring hospitalization or admission to the intensive care unit were recorded as major complications. Complications that did not comply with this definition were accepted as minor complications. Patients were kept under observation until they achieved preprocedural level of consciousness and acceptable vital parameters. Bronchial washing was performed for all patients. In patients with endobronchial lesions during bronchoscopy, biopsy was performed first, and then bronchial washing samples were taken. $10 \mathrm{~mL}$ isotonic $\mathrm{NaCl}$ liquid was administered to the bronchial orifice where the lesion was located, and then aspirated for bronchial washing or applied to the endobronchial lesion before and after the biopsy.

TNBs were performed by the interventional radiology department. Helical CT was used to guide biopsy needle placement. Patients received conscious sedation on request. Subcutaneous injection of $1 \%$ lidocaine was used for local anesthesia. All patients were kept under observation for 2 hours and underwent posteroanterior chest radiography after the biopsy procedures. Complications of TNB included pneumothorax, hemorrhage, tumor seeding along the needle biopsy tract, and air embolism. The patients who were hospitalized for pneumothorax and who had a chest tube were also examined.

The patients were divided into FB and TNB groups. A comparison was made between the two groups on the basis of lung cancer cell type. In addition, the role of bronchoscopic washing was investigated in patients who underwent FB with undiagnostic results. Complication rates of FB and TNB were evaluated.

\section{Ethics Committee Approval}

This study was approved by the Ethics Committee of the Umraniye Training and Research Hospital (Approval date: 18.12.2019, No: 239). Informed consent was waived because of the retrospective nature of the study.

\section{Statistical Analysis}

Descriptive analyses (frequency distributions, percentages) were used for statistical analysis of data analysis. Chi-squared analysis was used to analyze the frequency distribution of the data and the results were evaluated at a $95 \%$ confidence interval and $p<.05$ significance level. PSPP and Microsoft Excel programs were used for data analysis (PSPP is a free software; it can be redistributed and/or modified under the terms of the GNU General Public License as published by the Free Software Foundation; either version 3 of the License, or [at your option] any later version.).

\section{RESULTS}

The study included 182 lung cancer patients, out of whom 106 patients were diagnosed by FB and 76 patients were diagnosed by CT-guided TNB (Table I). No significant difference was observed between the diagnostic approaches for ADC $(p=.22)$. The diagnosis of SCLC and SCC was made more accurately by flexible bronchoscopy than by TNB and the differences were statistically significant ( $p=.01, p=.01$, respectively). Patients with LCC were diagnosed with only FB, whereas patients with undifferentiated carcinoma were diagnosed with only TNB. Broncho- 


\begin{tabular}{|c|c|c|c|}
\hline & \multicolumn{2}{|r|}{ Biopsy } & \multirow[b]{2}{*}{$p$} \\
\hline & FB $(N=106)$ & CT-guided TNB $(\mathrm{N}=76)$ & \\
\hline Adenocarcinoma & $34(32 \%)$ & $45(59.2 \%)$ & 0.22 \\
\hline Large cell carcinoma & $2(1.8 \%)$ & $0(0 \%)$ & - \\
\hline Small cell carcinoma & $22(20.7 \%)$ & $6(7.8 \%)$ & $0.01^{*}$ \\
\hline Squamous cell carcinoma & $48(45.2 \%)$ & $21(27.6 \%)$ & $0.01^{*}$ \\
\hline Undifferentiated carcinoma & $0(0 \%)$ & $4(5.2 \%)$ & - \\
\hline
\end{tabular}

TABLE 2. The contribution of bronchoscopic washing to the diagnosis of patients (N: 106) (\%) who underwent bronchoscopic biopsy

\begin{tabular}{ccccc} 
Bronchoscopic & Malignity & Bronchoscopic & Negative & $0(0 \%)$ \\
biopsy (N: I06) & negative (N:3) & washing & Positive & $3(2.8 \%)$ \\
& Malignity & Bronchoscopic & Negative & $51(48.1 \%)$ \\
positive (N: 103) & washing & Positive & $52(49 \%)$ \\
\hline
\end{tabular}

TABLE 3. Complications of flexible bronchoscopy (N: 106)

\begin{tabular}{|lcc|}
\hline Procedure-related complications & Hypoxemia & N(patient) (\%) \\
& Minor hemorrhage & $3(2.8 \%)$ \\
Anesthesia-related complications & Respiratory depression & $1(0.9 \%)$ \\
& Hypertension & $4(3.7 \%)$ \\
Total complication rate & & $12 / 106(11.3 \%)$ \\
\hline
\end{tabular}

TABLE 4. Transthoracic needle biopsy-related complications

\begin{tabular}{|llc|}
\hline Complications & Parenchymal Hemorrhage & $2(2.6 \%)$ \\
& Pneumothorax & II $(14.4 \%)$ \\
& $\begin{array}{l}\text { Parenchymal hemorrhage } \\
\text { and pneumothorax }\end{array}$ & $2(2.6 \%)$ \\
& Total complication rate & $15 / 76(19.7 \%)$ \\
\hline
\end{tabular}

scopic washing was found to be positive in 3 of the patients who could not be diagnosed with bronchoscopic biopsy (Table 2). Bronchoscopic washing was positive for malignancy in $49 \%$ of the patients who underwent bronchoscopic biopsy and had positive results. No major complications were detected after bronchoscopy (Table 3). Hypoxemia was detected in 4 patients who underwent bronchoscopy. Minor hemorrhage was observed in 3 patients. One patient had sedative drug-induced respiratory depression. Four patients had transient elevation of arterial blood pressure. No patients died after or during FB. All of the complications were treated medically. After FB, no patients required inpatient treatment or intensive care follow-up due to complications. The total minor complication rate of FB was found to be $11.3 \%$ when procedure- and anesthesia-related complications were evaluated together.

Pneumothorax was detected in $17 \%(\mathrm{~N}=13)$ of patients undergoing TNB (Table 4). When parenchymal hemorrhage and pneumothorax were evaluated together, the complication rate was $19.7 \%$. 8 out of 13 patients who developed pneumothorax required a chest tube. Patients who had pneumothorax and parenchymal hemorrhage and chest tube implantation were treated in hospital.

\section{DISCUSSION}

In this study, it was observed that SCLC and SCC were mostly diagnosed with FB. There was no significant difference between diagnostic methods for ADC. It was found that bronchial washings were positive in $51.8 \%$ of patients who underwent bronchoscopic biopsy and additionally diagnosed 3 patients with negative bronchoscopic biopsy. It has been observed that the complications of FB were less than TNB.

Lung cancer is a common cancer associated with high mortality rates. More than 2 million new lung cancer diagnoses were made, and 1.7 million people died due to lung cancer in 2018 (8). Lung cancer has been reported to be the cause in $14 \%$ of the newly diagnosed cancer patients and $20 \%$ of the deaths caused by cancer (8). Therefore, it is crucial to diagnose the disease without any delay and identify the stage to start an appropriate treatment immediately. There are different diagnostic tools for the diagnosis of lung cancer. FB and TNB are the most commonly used methods for the diagnosis of lung cancer. The size, localization of the lesion, relationship with patent airway, risk of complications in the patient, and current experience are important in the selection of biopsy method for the diagnosis of pulmonary nodules (9). Bronchoscopic techniques are preferred in central nodules located proximal to a patent bronchus, especially in patients at high risk of pneumothorax with TNB.

Lung tumors, depending on their location in the tracheobronchial tree, are categorized as central or peripheral. SCLC and SCC are usually centrally located and ADC and LCC are peripherally located tumors (7). As a general rule, FB is preferred for central lesions and TNB is preferred for peripheral lesions. The sensitivity of FB to detect malignancy in solitary pulmonary nodules depends on the size of the nodule, the proximity of the lesion to the bronchial tree, the presence of bronchus sign on CT, and the prevalence of cancer in the community (10). Malignancy detection rate of FB was found to be $75-94.5 \%$ in bronchoscopically visible lesions and $41.7 \%-81.2 \%$ in non-bronchoscopically visible lesions (II). Bronchoscopy can be used to perform bronchial washing, bronchial brushing, transbronchial needle aspiration biopsy (TBNA), and transbronchial biopsy (TBB) in addition to biopsy from endobronchial lesions (12). In the presence of an endobronchial lesion, the sensitivity of biopsy, bronchial brushing, and bronchial washing was reported to be $74 \%, 59 \%$, and $48 \%$, respectively (13). The diagnostic value of FB in bronchoscopically visible intramural tumors was reported to be about $55 \%$ and decreased further in cases where the tumor cannot be seen bronchoscopically (12). The diagnostic value of FB is approximately $88 \%$ with the combined use of bronchoscopic biopsy, bronchial washing, and brushing (I0).

This study reported that FB provided a more accurate diagnosis of SCC and SCLC than that observed with TNB. It was an expected outcome because both SCC and SCLC are centrally located tumors. There was no significant difference between bronchoscopy and TNB in the diagnosis of ADC. ADCs are known to be located peripherally and therefore, although it is expected to be diagnosed with a better accuracy by TNB, there was no difference between the two diagnostic procedures. Bronchial washing was positive in approximately half of the patients who underwent FB for lung cancer in this study. The 
diagnosis was made by bronchial washing in 3 patients who could not be diagnosed by bronchoscopic biopsy. These results showed the diagnostic contribution of bronchial washing in patients undergoing FB.

Complications related to FB have been reported to be between $0.1 \%$ and $11 \%$ and the procedure-related mortality rate to be between $0 \%$ and $0.1 \%$ (14). The main reason for the differences in complication rates is related to the definition of complications in the studies. A systematic analysis of the complications of FB was performed (6). Severe complications were rare and the rate of pneumothorax requiring intervention was $0 \%-2.1 \%$ after transbronchial biopsies (6). Oxygen desaturation was found in $0.7 \%-76.3 \%$ of the patients undergoing $\mathrm{FB}$ and bleeding was found in $2.5 \%-89.9 \%$ of the patients undergoing FB. In general, FB was concluded to be a safer procedure, but a consensus on the identification of complications is required. Approach to patients with comorbidity in patient selection prior to FB is very important. Particular attention is required in patients with concomitant airway obstructive pulmonary diseases such as chronic obstructive lung disease and bronchial asthma (I5). In a prospective study, actual blood loss and factors that may cause bleeding were investigated in 234 patients undergoing FB (16). The results reported mild bleeding $(5-20 \mathrm{~mL})$ in 19 patients, moderate bleeding $(20-100 \mathrm{~mL}$ ) in 5 patients, and no severe bleeding $(>100 \mathrm{~mL})$ in any patient (16). In another study where FB was performed in 400 patients without comorbid diseases, complications (bleeding, pneumothorax, collapse, and infection) were detected in $3.25 \%$ of the patients (17). The low complication rates may be due to the exclusion of patients with comorbidity. In this study, no serious major complications because of FB were detected. Minor complications occurred in $11.3 \%$ of the patients, but none of them required hospitalization and were easily treatable. Differences reported in studies examining bronchoscopic complications may be due to the definition of complications. In this study, FB was found to be a safer diagnostic procedure.

TNB is preferred primarily in patients with peripheral pulmonary nodules, and approximately $90 \%$ of patients can be diagnosed with TNB. Although the diagnostic value of TNB is high, its complications are not uncommon, and they increase the risk of morbidity and mortality. Pneumothorax was detected in $25 \%$ of the patients who underwent TNB and $15 \%$ of them required chest tube treatment (3). The complication rate of TNB in this study was similar to that reported by other studies. When hemorrhage was included, the overall complication rate was $19.73 \%$. The patients who developed pneumothorax required hospitalization and approximately half of these patients underwent tube thoracostomy. TNB is not a suitable choice in patients with previous pneumothorax and emphysema in the lung parenchyma (18). The positive predictive factors of TNB in the diagnosis of pulmonary nodules are related to nodule size (larger diameter, better accuracy), noncalcified density (better accuracy as density increases), and the distance between nodule and pleura (19). In a meta-analysis, TNB results were not diagnostic in $6.8 \%$ of patients who underwent TNB biopsy (20). 59.3\% of the patients with non-diagnostic TNB were diagnosed with malignancy (20). These results suggest that further diagnostic procedures are required or TNB may be repeated in patients with negative TNB biopsy.
In this study, it was observed that bronchoscopic complications were less than those reported with TNB and did not require additional therapeutic treatment or hospitalization. In contrast, TNB complications were relatively more severe, requiring tube thoracostomy and hospitalization.

This study had some limitations. First, this study was conducted retrospectively. The presence or absence of endobronchial lesions in patients diagnosed with bronchoscopy has not been recorded separately. Furthermore, the localization of the lesions in the lungs has not been studied.

The study concluded that SCLC and SCC were more accurately diagnosed with FB than with TNB. Bronchoscopic washing helped in complementing the diagnosis of the types of cancers studied. FB was found to be much safer and associated with lower complication rates as compared with CT-guided TNB.

Ethics Committee Approval: Ethics committee approval was received for this study from the ethics committee of Umraniye Training and Research Hospital (Approval date: 18. 12. 2019, No: 239).

\section{Informed Consent: N/A}

Peer-review: Externally peer-reviewed.

Author contributions: Concept - T.C., K.C.; Design - T.C., K.C., O.A.; Supervision - O.O., Z.K.; Resource - K.C., O.A.; Materials - K.C., T.C.; Data Collection and/or Processing - K.C., O.A.; Analysis and/or Interpretation - O.O., Z.K.; Literature Search - O.A., K.C.; Writing - T.C., K.C., O.A.; Critical Reviews -O.O., Z.K.

Conflict of Interest: Authors have no conflicts of interest to declare.

Financial Disclosure: The authors declared that this study has received no financial support.

\section{REFERENCES}

I. Fitzmaurice C, Dicker D, Pain A, Hamavid H, Moradi-Lakeh M, MacIntyre MF, et al. The Global Burden of Cancer 2013. JAMA Oncol 20I5; I(4): 505-27. [Crossref]

2. Wiener RS, Schwartz LM, Woloshin S, Welch HG. Population-based risk for complications after transthoracic needle lung biopsy of a pulmonary nodule: an analysis of discharge records. Ann Intern Med 20II; 155(3): 137-44. [Crossref]

3. Kosar F, Caliskan T. Bronchoscopic Diagnosis of Pulmonary Nodules. Turkiye Klinikleri J Pulm Med-Special Topics 20I8; II(I): 26-32.

4. Baaklini WA, Reinoso MA, Gorin AB, Sharafkaneh A, Manian P. Diagnostic yield of fiberoptic bronchoscopy in evaluating solitary pulmonary nodules. Chest 2000; II7(4): 1049-54.[Crossref]

5. Chan EY, Gaur P, Ge Y, Kopas L, Santacruz JF, Gupta N, et al. Management of the Solitary Pulmonary Nodule. Arch Pathol Lab Med 2017; 14I(7): 927-3I. [Crossref]

6. Leiten EO, Martinsen EM, Bakke PS, Eagan TM, Grønseth R. Complications and discomfort of bronchoscopy: a systematic review. Eur Clin Respir J 2016; 3: 33324. [Crossref]

7. Travis WD. Pathology of lung cancer. Clin Chest Med 20II; 32(4): 66992. [Crossref]

8. Miranda-Filho A, Piñeros M, Bray F. The descriptive epidemiology of lung cancer and tobacco control: a global overview 2018. Salud Publica Mex 2019; 6I(3): 219-29. [Crossref]

9. Gould MK, Donington J, Lynch WR, Mazzone PJ, Midthun DE, Naidich DP, et al. Evaluation of individuals with pulmonary nodules: when is it lung cancer? Diagnosis and management of lung cancer, 3rd ed: American College of Chest Physicians evidence-based 
clinical practice guidelines. Chest 2013; 143(5 Suppl): e93S-el20S. [Crossref]

10. Lee $\mathrm{P}, \mathrm{Colt} \mathrm{HG}$. Bronchoscopy in lung cancer: appraisal of current technology and for the future. J Thorac Oncol 20I0; 5(8): 1290-300. [Crossref]

II. Slade MG, Rahman NM, Stanton AE, Curry L, Slade GC, Clelland $\mathrm{CA}$, et al. Improving standards in flexible bronchoscopy for lung cancer. Eur Respir J 20II; 37(4): 895-90I. [Crossref]

12. Herth FJ, Eberhardt R. Flexible bronchoscopy and its role in the staging of non-small cell lung cancer. Clin Chest Med 20I0; 3I(I): 87100. [Crossref]

13. Tsim S, O'Dowd CA, Milroy R, Davidson S. Staging of non-small cell lung cancer (NSCLC): a review. Respir Med 2010; 104(12): 1767-74. [Crossref]

14. Stahl DL, Richard KM, Papadimos TJ. Complications of bronchoscopy: A concise synopsis. Int J Crit IIIn Inj Sci 2015; 5(3): 189-95. [Crossref]

15. Asaad A, Clum S, Rumbak M. Fiberoptic Bronchoscopy Complications. J Respir Med Lung Dis 2017; 2(4): 1025.
16. Carr IM, Kogelenberg CF, von Groote-Bidlingmaier F, Mowlana A, Silos K, Haverman T, et al. Blood loss during flexible bronchoscopy: a prospective observational study. Respiration 2012; 84(4): 312-8. [Crossref]

17. Fazlalizadeh H, Adimi P, Kiani A, et al. Evaluation of bronchoscopy complications in a tertiary health care center. Tanaffos 20I4; I3(4): 48-50.

18. Wiener RS, Schwartz LM, Woloshin S, Welch HG. Population-based risk for complications after transthoracic needle lung biopsy of a pulmonary nodule: an analysis of discharge records. Ann Intern Med 20Il; 155(3): 137-44. [Crossref]

19. Yang W, Jiang H, Khan AN, Allen C, Bertolaccini L, Lv T, et al; written on behalf of the AME Lung Cancer Collaborative Group. Transthoracic needle aspiration in solitary pulmonary nodule. Transl Lung Cancer Res 2017; 6(I): 76-85. [Crossref]

20. Chae KJ, Hong H, Yoon SH, Hahn S, Jin GY, Park CM, et al. Non-diagnostic Results of Percutaneous Transthoracic Needle Biopsy: A Meta-analysis. Sci Rep 2019; 9(1): 12428. [Crossref] 